\title{
Identification of ITPR1 as a hub gene of Group 3 Medulloblastoma and coregulated genes with potential prognostic values
}

Pablo Ferreira das Chagas ( $\sim$ pablochagas@usp.br)

University of Sao Paulo Faculty of Medicine of Ribeirao Preto: Universidade de Sao Paulo Faculdade de Medicina de Ribeirao Preto https://orcid.org/0000-0002-0652-728X

\section{Graziella Ribeiro de Sousa}

University of Sao Paulo Faculty of Medicine of Ribeirao Preto: Universidade de Sao Paulo Faculdade de Medicina de Ribeirao Preto

\section{Luciana Chain Veronez}

University of Sao Paulo Faculty of Medicine of Ribeirao Preto: Universidade de Sao Paulo Faculdade de Medicina de Ribeirao Preto

\section{Andrea Martins-da-Silva}

University of Sao Paulo Faculty of Medicine of Ribeirao Preto: Universidade de Sao Paulo Faculdade de Medicina de Ribeirao Preto

\section{Carolina Alves Pereira Corrêa}

University of Sao Paulo Faculty of Medicine of Ribeirao Preto: Universidade de Sao Paulo Faculdade de Medicina de Ribeirao Preto

\section{Gustavo Alencastro Veiga Cruzeiro}

Harvard Medical School

\section{Luis Fernando Peinado Nagano}

University of Sao Paulo Faculty of Medicine of Ribeirao Preto: Universidade de Sao Paulo Faculdade de Medicina de Ribeirao Preto

\section{Rosane Gomes de Paula Queiroz}

University of Sao Paulo Faculty of Medicine of Ribeirao Preto: Universidade de Sao Paulo Faculdade de Medicina de Ribeirao Preto

\section{Suely Kazue Nagahashi Marie}

Sao Paulo University Faculty of Medicine: Universidade de Sao Paulo Faculdade de Medicina

\section{Silvia Regina Brandalise}

Boldrini Children's Center

\section{Carlos Alberto Scrideli}

University of Sao Paulo Faculty of Medicine of Ribeirao Preto: Universidade de Sao Paulo Faculdade de Medicina de Ribeirao Preto

\section{Luiz Gonzaga Tone}


University of Sao Paulo Faculty of Medicine of Ribeirao Preto: Universidade de Sao Paulo Faculdade de Medicina de Ribeirao Preto

\section{Elvis Terci Valera}

University of Sao Paulo Faculty of Medicine of Ribeirao Preto: Universidade de Sao Paulo Faculdade de Medicina de Ribeirao Preto

\section{Research Article}

Keywords: Grp3-MB, Bioinformatic analysis, ITPR1, Metastasis, RNA-Seq

Posted Date: October 28th, 2021

DOl: https://doi.org/10.21203/rs.3.rs-899456/v1

License: (1) This work is licensed under a Creative Commons Attribution 4.0 International License. Read Full License

Version of Record: A version of this preprint was published at Journal of Molecular Neuroscience on November 25th, 2021. See the published version at https://doi.org/10.1007/s12031-021-01942-3. 


\section{Abstract}

The Group 3 Medulloblastoma ( Grp3-MB ) is an aggressive molecular subtype with a high incidence of metastasis and deaths. In this study, were used an RNA sequencing data (RNA-Seq ) from a Brazinian cohort of MBs to identify hub genes associated with the metastatic risk. Data validation were performed by using multiple large datasets from MBs (GSE85217, GSE37418, EGAS00001001953). DESeq2 package in R software was used to identify the differentially expressed genes ( DEGs) in our RNA-Seq data. The DEGs data were accessed to construct the modules/graphs of coexpression and to identify hub genes through Cytoscape platform. The co-regulated genes were enriched by the Kyoto Encyclopedia of Genes and Genomes (KEGG) pathway and the Protein-protein interaction ( PPI) network was visualized by Cytoscape. The Kaplan-Meier plotter and ROC curves were used to validate the diagnostic and prognostic values of specific biomarkers identified through this model. We identified that Inositol 1,4,5triphosphate receptor type 1 (ITPR1) as a downregulated hub gene, with a high diagnostic accuracy to Grp3-MBs and associated with tumor metastasis. In addition, we identified genes significantly correlated with ITPR1 that were associated with metastasis in Grp3-MB ( ATP1A2, MTTL7A and RGL1), and worst overall survival in MBs ( ANTXR1 and RGL1). Our findings suggest that the ITPR1 hub gene is potentially involved in the metastatic process for Grp3-MB. Our data also provide evidence of targets that may serve as prognostic predictors and/or regulators for the metastatic process that maybe explored for further research of individualized therapy to Grp3-MBs.

\section{Introduction}

Medulloblastoma (MB) is the most common malignant embryonal brain tumor in the pediatric population (Northcott et al. 2019). The current consensus established by the World Health Organization (WHO) recognizes four main molecular and clinico-pathological distinct MB subtypes: Wingless-activated (WNT-MB), Sonic Hedgehog-activated (SHH-MB), and non-WNT/non-SHH MBs: Group 3 (Grp3-MB), and Group 4 (Grp4-MB) (Louis et al. 2021). Grp3-MBs represent approximately $25 \%$ of all MB cases, being the deadliest across molecular subgroups and frequently (15-20\%) showing metastasis at diagnosis in infants and older children (Cavalli et al. 2017; Juraschka and Taylor 2019).

Next Generation Sequencing (NGS) techniques such as RNA Sequencing (RNA-Seq) and robust bioinformatics analysis have been used over the years to uncover potential biomarkers in MBs (Guo et al. 2020b). Although integrative genomic studies have clarified several mechanisms on MBs tumorigenesis, (Juraschka and Taylor 2019; Menyhárt and Győrffy 2019) it is of note the paucity of genes with clinical relevance that ultimately could be used as metastastatic biomarkers and predictors for prognostication, improving therapeutic strategies for Grp3-MB.

In this study, we identified the Inositol 1,4,5-trisphosphate receptor 1 (ITPR1) gene, a regulator of calcium signaling $\left(\mathrm{Ca}^{2+}\right)$, homeostasis and autophagy in cancer, (Gambardella et al. 2020; Sneyers et al. 2020) as a hub gene for Grp3-MBs. In addition, we showed that ITPR1 is specifically downregulated with a high diagnostic accuracy to Grp3-MB in our and multiple independent cohorts of MB. Interestingly, the low 
expression of ITPR1 was associated with the presence of metastasis in MBs. In addition, we identified 17 genes significantly coregulated with ITPR1, highlighting the ATP1A2, MTTL 7A and RGL 1 which demonstrated association with metastasis in Grp3-MB, and ANTXR1 and RGL 1 correlated to worse overall survival (OS) in MBs. Our study provides for the first-time insights about the association of ITPR1 as a prognostic biomarker and a potential therapeutic target to Grp3-MBs.

\section{Materials And Methods}

\section{RNA-Sequencing from a data generated in-house cohort}

In a previous study of our group, 17 pediatric MB samples from Brazilian patients were sequenced and deposited in the GEO database under there Cord number GSE181293. The RNA-Seq data of our in-house cohort was used to identify the differentially expressed genes (DEGs) and also to gain insights on hub genes for Grp3-MBs as follows.

\section{Co-expression network and Hub Gene selection}

DESeq2 package in R software (version 3.4.3) was used to identify the DEGs into our RNA-Seq data (Love et al. 2014). The counts expression data (for Grp3-MB) were normalized and used as input for network construction in CEMiTool (followed script standard), (Russo et al. 2018) which DEGs with similar expression patterns were grouped into modules via the average linkage hierarchical clustering. Within the networks of coexpression were identified the genes that have the highest number of connections and/or interactions, using the connectivity module measured by the absolute value of the Pearson correlation coefficient $>0.5$ and the graphs were generated to represent interacting genes (hub genes) through the extension called GeneMania (Montojo et al. 2010) in the Cytoscape 3.7.1 platform (https://cytoscape.org/). We screened genes closely linked in the modules and assessed the correlation of these genes' expression value with presence of metastasis using the clinical data of the pediatric MBs GSE85217 ( $n=643$ ) (Cavalli et al. 2017), that were downloaded from R2 Platform (Genomics Analysis and Visualization Platform - http://r2.amc.nl). We used the Student's t-test (non-parametric Mann Whitney test) and a p-value $\leq 0.05$ was considered as statistically significant. Thus, the ITPR 1 hub gene was retained for further analysis.

\section{Evaluation of ITPR1 expression and identification of co-regulated genes in multiple pediatric MBs retrospective cohorts}

Different datasets were downloaded from R2 Platform (Genomics Analysis and Visualization Platform - http://r2.amc.nl) to evaluate ITPR1 expression levels. Two out of three datasets were derived of microarray data: GSE85217 ( $n=643$ ) (Cavalli et al. 2017); GSE37418 ( $n=73)$ (Robinson et al. 2012) and one of RNA-Seq data: EGAS00001001953 ( $\mathrm{n}=186)$ (Northcott et al. 2017). ITPR1-coregulated genes were identified using common elements of all four MB datasets with a p-value $<0.05$ and a Pearson's correlation coefficient $>0.5$. Further, a Venn diagram was performed to overlap coregulated genes with ITPR1 in all cohorts (data not shown). To better visualize the profile expression of ITPR1-coregulated 
genes we generated a heatmap using the Complex Heatmap package (Gu et al. 2016) with pre-set metrics as Pearson's correlation distance parameter and the algorithm WardD2.

\section{Protein-protein interaction (PPI) network}

The Protein-protein interactions (PPI) among the commom ITPRT-coregulated genes were evaluated using the Search Tool for the Retrieval of Interacting Genes (STRING) database (http://www.stringdb.org/) (Szklarczyk et al. 2015). Validated interactions with a combined score $>0.9$ were selected as significant (Bozhilova et al. 2019) Then, integration of PPI networks was constructed using the Cytoscape software (https://cytoscape.org) (Shannon et al. 2003) with GeneMANIA plugin to identify biological network integration for gene prioritization (Warde-Farley et al. 2010).

\section{KEGG pathways enrichment analysis}

We conducted Kyoto Encyclopedia of Genes and Genomes (KEGG) pathway enrichment analysis to better understand the putative functions of ITPR1-coregulated genes using the Enrichr database (https://maayanlab.cloud/Enrichr/) (Kanehisa and Goto, 2000). A p-value of $<0.05$ was considered to represent a statistically significant enrichment of DEGs in pathway.

\section{Survival and metastasis analysis}

Kaplan-Meier curves and the long-rank tests were performed to evaluate overall survival (OS) according to the expression of the ITPR1-coregulated genes using the GSE85217 dataset (Cavalli et al. 2017). This dataset was preferred to this analysis due to the higher number of MB cases available to be analysed, along with better clinical data associated to genetic dataset. Patients were divided into two groups (low vs. high) according to the median expression of each gene. First, we considered the data from all subtypes of MBs samples primary $(n=343)$ and metastasis $(n=167)$. Then, we used only data of Grp3MBs primary samples $(n=62)$ and metastasis $(n=43)$ information.

\section{Statistical analysis}

We performed statistical analysis comparing expressions among two different groups by Student's t-test (non-parametric Mann Whitney test). Analyzes between more the two groups were used One-way-ANOVA non-parametric followed Tukey post test. Receiver operating characteristic (ROC) curves were used to evaluate the discrimination of ITPR1 expression levels into molecular MBs subtypes. The accuracy was determined by the area under the curve (AUC). Graphs were generated with GraphPad Prism 8.0 (GraphPad Software, San Diego, CA, USA), considering $p$-value $<0.05$ as statistically significant.

\section{Results}

RNA-Seq analysis reveals the ITPR1 as a hub gene in our pediatric cohort of MB 
In order to identify key genes in Grp3-MB tumor we first identified DEGs in Grp3-MB compared to other molecular subtypes (WNT-MB, SHH-MB and Grp4-MB) in our cohort. Next, co-expression network analysis identified a total of five specific key modules related to Grp3-MB (Supplementary Fig. 1). In one out of these modules, we identified ITPR1 as a hub gene (Fig. 1). Previously, the ITPR1 was found downregulated in Grp3-MBs highlighting a novel and promising therapeutic target (Beckmann et al. 2019; Castillo-Rodríguez et al. 2018). Thus, we selected the ITPR1 as an interesting gene related with tumor biology for subsequent analysis given that ITPR 1 into Grp3-MBs carcinogenesis are poorly understood to date.

\section{The low expression of ITPR1 gene is associated with metastasis and represents a potential biomarker in Grp3/Grp4-MBs}

In our cohort, we showed that low expression gene of ITPR1 in Grp3-MBs when compared to other MBs subtypes (Fig 2a). Thus, the expression profile of ITPR 1 was also evaluated in three additional independent databases GSE85217 (Fig. 2b), GSE37418 (Fig. 2C), and EGAS00001001953 (Fig. 2d) of MBs. Interestingly, it was possible to validate a specific low expression profile of ITPR1 in Grp3-MBs or Grp4-MBs compared to WNT and SHH-MBs.

Once we observed that ITPR1 is downregulated in both Grp3 and Grp4-MBs, we evaluted its potential as a biomarker of these two specific subgroups. ROC analysis showed that the AUC was 0.846 , suggesting a high diagnostic accuracy in the discrimination between Grp3/Grp4-MBs from non-Grp3/Grp4-MBs (Fig. 2e). Remarkably, the lower expression of ITPR1 was also assoaciated with the presence of metastasis in MB (Fig. 2f). Together, these results suggest that the low expression of ITPR 1 gene is a potential biomarker of Grp3/Grp4-MBs and is potentially related to metastasis process in this setting.

\section{ITPR1-coregulated genes potentially regulate pathways related to metastasis in MBs}

To gain further insights into the putative functions of the hub gene ITPR1, we identified genes significantly coregulated with ITPR1 expression exploring data of three MBs public databases (GSE85217, GSE37418, EGAS00001001953) and our RNA-Seq data. A total of 17 genes were shown to be commonly coregulated with ITPR 1 in all four datasets, which roles previously described in cancer are summarized in Supplementary Table 1. Interestingly, the expression profile of these 17 ITPR1-coregulated genes in four datasets converges to a low expression of these genes to Grp3-MBs in the most of samples, as shown in Fig. 3a-d. The PPI network and KEGG enrichment analysis revelead that the seventeen ITPR1coregulated genes present genetic and co-expression interactions (Fig. 4a) and are significantly associated with the signaling pathways regulating pluripotency of stem cells, regulation of actin cytoskeleton, Ras signaling pathway and cell tigh-junction (Fig. 4b). The genes that are involved into each correlated pathway are described in Supplementary Table 2. Altogether, our results indicate an important regulatory network between ITPR1 and co-regulated genes that potentially are involved into a signaling net associated with metastatic processes in MBs. 
To evaluate the prognostic value and clinical relevance of the 17 ITPR1-coregulated genes we performed association and survival analysis using the GSE85217 dataset (Cavalli et al. 2017). Interestingly, the low expression of all the 17 genes was significantly associated with the ocurrance of metastasis when analyzed in all MBs datasets ( $<0.001)$ Supplementary Fig. 2). In addition, we observed that the expression of three out of 17 common-genes, the ATP1A2, MTTL 7A and RGL 1 were also significantly associated with presence of metastasis specifically in Grp3-MBs patients ( $\mathrm{p}<0.001)$ (Fig. 5a-c). Regarding prognostic value of the 17 ITPR1-coregulated genes, lower expression levels of ANTXR1 and RGL 1 were significantly associated with worse OS in MBs ( $p=0.0037$ and $p=0.029$, respectively- Fig. $5 d-e)$. Collectively, our findings suggest that ITPR 1 and coregulated genes are potential biomarkes for of poor prognosis and metastasis in MBs.

\section{Discussion}

a

"Omics" studies (e.g., exome/proteomics/single-cell approaches) and integrative large NGS data analysis are valuable tools to identify novel biomarkers in cancer. Although many studies regarding biomarkers for MBs have been published (Huang et al. 2020), a sharper identification of hub genes and their interactions can be very helpful to unravel molecular mechanism associated with prognosis and metastasis, particularly for Grp3-MBs.

In the current study, we demonstrated that ITPR1 is a hub gene downregulated in Grp3-MBs and associated to metastasis. The ITPR1 gene is highly expressed in the Purkinje cells in the central nervous system (SNC) and the modulation of intracellular calcium homeostasis is the main biological function of this gene (Foskett et al. 2007; Nakanishi et al. 1991). Recently, the ITPR1 has been reported to present a complex role in cancer dynamics by controlling cell survival and cell death via regulation of apoptosis and autophagy (Parys et al. 2021). This is especially relevant, given that programmed cell death through autophagy plays crucial role in progression and metastasis in cancer (Su et al. 2015) and the low expression of autophagy-related genes is associated with poor prognosis and tumor progression (Wang et al. 2015a; Xu et al. 2019). Based on these reports and in our results, we suggest that the downregulation of ITPR 1 is involved in the crosstalk between reduced autophagy and increased metastatic mechanisms and may play an important role towards a malignant phenotype in Grp3-MBs. Yet, further studies are needed to test and to explore this hypothesis.

Previously, the ITPR1 was found downregulated in Grp3/Grp4-MBs highlighting a novel and promising therapeutic target. Meanwhile, this data was not explored the underlying molecular mechanisms of the ITPR1 into Grp3/Grp4-MBs carcinogenesis (Beckmann et al. 2019; Castillo-Rodríguez et al. 2018). We screened the gene expression profile of ITPR1 among our and others three pediatric MBs dataset: GSE85217 (Cavalli et al. 2017); GSE37418 (Robinson et al. 2012) and EGAS00001001953 (Northcott et al. 2017), and the low expression of ITPR1 gene was also found on Grp3/Grp4-MBs when compared to WNT/SHH-MBs. Of note, our ROC analysis showed that low expression of ITPR1 gene is a potential predictive biomarker for segregating Grp3/Grp4-MBs subtypes. 
Furthermore, by screening the degree of nodes in the PPI network and KEGG enrichment analysis, we showed that overlapping of these ITPR1-coregulated genes present genetic and co-expression interactions and were significantly associated with the signaling pathways regulating pluripotency of stem cells, regulation of actin cytoskeleton, Ras signaling pathway and tigh junction. The Ras Signaling was reported as regulator key element of several aspects of normal cell growth and malignant transformation. Further, it was also associated with metastatic dissemination and poor survival in breast cancer (Wright et al. 2015). Surprisingly, we showed that ITPR1-coregulated genes are also involved in relevant hallmarks of cancer as metastasis, cell invasion, migration, diferention and proliferation, as described in Table 1. Intriguingly, we also observed that in most of the samples of pediatric MBs tissues from all four cohorts, 17 ITPR1-coregulated genes were also found downregulated in the majority of samples of Grp3-MBs. Together, these ITPR1-coregulated genes are involved in pathways associated to the metastatic markers of Grp3-MBs.

Finally, we also found that low gene expression of ATP1A2, MTTL 7A and RGL 1 were significantly associated with the ocurrance of metastasis, particularly for Grp3-MBs. In addition, we demonstrated that low gene expression of $R G L 1$ was associated with worse OS in MBs. Studies addressing the role of these genes in tumorigenic processes also are very scarce. Interestingly, the low gene expression of ATP1A2 was also found in breast cancer as a predictive and a prognostic factor (Bogdanov et al. 2017). Yet, the association between the downregulation of RGL 1, metastasis and worse OS on MBs were neither described, nor explored to date.

\section{Conclusion}

In summary, this study highlights ITPR1 as a hub gene for Grp3-MB. Using an integrative bioinformatic approach, ITPR1 is potentially involved in the metastatic process of MBs. Nevertheless, further investigations are needed to fully verify and validate the function of this gene in MB setting, and to balance their role in the underlying mechanisms of Grp3-MB metastization.

\section{Ethical Statement}

Ethics approval and consent to participate: This study was approved by the HC/FMRP-USP Research Ethics Committee (Approval number: CAAE: 62604816.4.000.5440) and informed consent was obtained from all patients included.

Consent for publication: Not applicable.

Availability of data and materials: The data and other items supporting the results of the study will be made available upon reasonable request.

Competing interests: The authors declare that they have no competing interests. 
Funding: The study was supported by public Brazilian grants from the São Paulo State Research Foundation (FAPESP), Grant numbers: 2014/20341-0, 2017/26160-5, Brazilian Research Council (CNPq), Coordination for the Improvement of Higher Education Personnel (CAPES) Financial Code 001 and Foundation for Support of Education, Research and Assistance (FAEPA), Brazil

Authors' contributions: E.T.V. and P.F.C planned and conducted data analysis and drafted the manuscript. G.R.S, L.C.V, A.M.S, C.A.P.C; G.A.V.C and L.F.P.N wrote and organized the data, created the figures/tables, edited and critically revised the manuscript. R.G.P.Q, S.K.N.M, S.R.B, C.A.S and L.G.T revised the text for important intellectual content. All authors critically read and approved the final manuscript

Acknowledgements: We want to thank and acknowledge patients and families affected by medulloblastomas for their generous contributions to these studies.

\section{References}

Beckmann PJ, Larson JD, Larsson AT, et al (2019) Sleeping Beauty Insertional Mutagenesis Reveals Important Genetic Drivers of Central Nervous System Embryonal Tumors. Cancer Res 79:905-917. https://doi.org/10.1158/0008-5472.CAN-18-1261

Bogdanov A, Moiseenko F V., Dubina M (2017) Abnormal expression of ATP1A1 and ATP1A2 in breast cancer. F1000Research 6:10. https://doi.org/10.12688/f1000research.10481.1

Bozhilova L V., Whitmore A V., Wray J, et al (2019) Measuring rank robustness in scored protein interaction networks. BMC Bioinformatics 20:446. https://doi.org/10.1186/s12859-019-3036-6

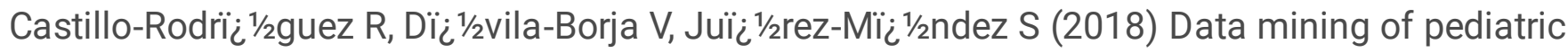
medulloblastoma microarray expression reveals a novel potential subdivision of the Group 4 molecular subgroup. Oncol Lett. https://doi.org/10.3892/ol.2018.8094

Cavalli FMG, Remke M, Rampasek L, et al (2017) Intertumoral Heterogeneity within Medulloblastoma Subgroups. Cancer Cell 31:737-754.e6. https://doi.org/10.1016/j.ccell.2017.05.005

Chen D, Bhat-Nakshatri P, Goswami C, et al (2013) ANTXR1, a Stem Cell-Enriched Functional Biomarker, Connects Collagen Signaling to Cancer Stem-like Cells and Metastasis in Breast Cancer. Cancer Res 73:5821-5833. https://doi.org/10.1158/0008-5472.CAN-13-1080

Chen G, Sun L, Han J, et al (2019) RILPL2 regulates breast cancer proliferation, metastasis, and chemoresistance via the TUBB3/PTEN pathway. Am J Cancer Res 9:1583-1606

Choi SA, Lee JY, Phi JH, et al (2014) Identification of brain tumour initiating cells using the stem cell marker aldehyde dehydrogenase. Eur J Cancer 50:137-149. https://doi.org/10.1016/j.ejca.2013.09.004

Foskett JK, White C, Cheung K-H, Mak D-OD (2007) Inositol Trisphosphate Receptor Ca 2+ Release Channels. Physiol Rev 87:593-658. https://doi.org/10.1152/physrev.00035.2006 
Fukuhisa $\mathrm{H}$, Seki N, Idichi T, et al (2019) Gene regulation by antitumor miR-130b-5p in pancreatic ductal adenocarcinoma: the clinical significance of oncogenic EPS8. J Hum Genet 64:521-534. https://doi.org/10.1038/s10038-019-0584-6

Gambardella J, Lombardi A, Morelli MB, et al (2020) Inositol 1,4,5-Trisphosphate Receptors in Human Disease: A Comprehensive Update. J Clin Med 9:1096. https://doi.org/10.3390/jcm9041096

Gu Z, Eils R, Schlesner M (2016) Complex heatmaps reveal patterns and correlations in multidimensional genomic data. Bioinformatics 32:2847-9. https://doi.org/10.1093/bioinformatics/btw313

Guo X, Piao H, Zhang Y, et al (2020a) Overexpression of microRNA-129-5p in glioblastoma inhibits cell proliferation, migration, and colony-forming ability by targeting ZFP36L1. Bosn J Basic Med Sci. https://doi.org/10.17305/bjbms.2019.4503

Guo Y, Huang P, Ning W, et al (2020b) Identification of Core Genes and Pathways in Medulloblastoma by Integrated Bioinformatics Analysis. J Mol Neurosci 70:1702-1712. https://doi.org/10.1007/s12031-02001556-1

Hu G, Wang R, Wei B, et al (2020) Prognostic Markers Identification in Glioma by Gene Expression Profile Analysis. J Comput Biol 27:81-90. https://doi.org/10.1089/cmb.2019.0217

Huang P, Guo Y-D, Zhang H-W (2020) Identification of Hub Genes in Pediatric Medulloblastoma by Multiple-Microarray Analysis. J Mol Neurosci 70:522-531. https://doi.org/10.1007/s12031-019-01451-4 Joy A, Moffet J, Neary K, et al (1997) Nuclear accumulation of FGF-2 is associated with proliferation of human astrocytes and glioma cells. Oncogene 14:171-183. https://doi.org/10.1038/sj.onc.1200823 Juraschka K, Taylor MD (2019) Medulloblastoma in the age of molecular subgroups: a review. J Neurosurg Pediatr 24:353-363. https://doi.org/10.3171/2019.5.PEDS18381

Kanehisa M (2000) KEGG: Kyoto Encyclopedia of Genes and Genomes. Nucleic Acids Res 28:27-30. https://doi.org/10.1093/nar/28.1.27

Key J, Mueller AK, Gispert S, et al (2019) Ubiquitylome profiling of Parkin-null brain reveals dysregulation of calcium homeostasis factors ATP1A2, Hippocalcin and GNA11, reflected by altered firing of noradrenergic neurons. Neurobiol Dis 127:114-130. https://doi.org/10.1016/j.nbd.2019.02.008

Li Y, Xiao X, Bossé Y, et al (2019) Genetic interaction analysis among oncogenesis-related genes revealed novel genes and networks in lung cancer development. Oncotarget 10:1760-1774. https://doi.org/10.18632/oncotarget.26678

Louis DN, Perry A, Wesseling P, et al (2021) The 2021 WHO Classification of Tumors of the Central Nervous System: a summary. Neuro Oncol 23:1231-1251. https://doi.org/10.1093/neuonc/noab106 
Love MI, Huber W, Anders S (2014) Moderated estimation of fold change and dispersion for RNA-seq data with DESeq2. Genome Biol. https://doi.org/10.1186/s13059-014-0550-8

McKinnon CM, Mellor H (2017) The tumor suppressor RhoBTB1 controls Golgi integrity and breast cancer cell invasion through METTL7B. BMC Cancer 17:145. https://doi.org/10.1186/s12885-017-3138-3

Menyhárt O, Győrffy B (2019) Principles of tumorigenesis and emerging molecular drivers of $<\mathrm{scp}>\mathrm{SHH}</ \mathrm{scp}>$-activated medulloblastomas. Ann Clin Transl Neurol 6:990-1005.

https://doi.org/10.1002/acn3.762

Montojo J, Zuberi K, Rodriguez H, et al (2010) GeneMANIA cytoscape plugin: Fast gene function predictions on the desktop. Bioinformatics. https://doi.org/10.1093/bioinformatics/btq562

Nakanishi S, Maeda N, Mikoshiba K (1991) Immunohistochemical localization of an inositol 1,4,5trisphosphate receptor, P400, in neural tissue: studies in developing and adult mouse brain. J Neurosci 11:2075-2086. https://doi.org/10.1523/JNEUROSCl.11-07-02075.1991

Northcott PA, Buchhalter I, Morrissy AS, et al (2017) The whole-genome landscape of medulloblastoma subtypes. Nature 547:311-317. https://doi.org/10.1038/nature22973

Northcott PA, Robinson GW, Kratz CP, et al (2019) Medulloblastoma. Nat Rev Dis Prim 5:11. https://doi.org/10.1038/s41572-019-0063-6

Parys JB, Bultynck G, Vervliet T (2021) IP3 Receptor Biology and Endoplasmic Reticulum Calcium Dynamics in Cancer. pp 215-237

Politis PK, Akrivou S, Hurel C, et al (2008) BM88/Cend1 is involved in histone deacetylase inhibitionmediated growth arrest and differentiation of neuroblastoma cells. FEBS Lett 582:741-748. https://doi.org/10.1016/j.febslet.2008.01.052

Robinson G, Parker M, Kranenburg TA, et al (2012) Novel mutations target distinct subgroups of medulloblastoma. Nature 488:43-48. https://doi.org/10.1038/nature1121

Russo PST, Ferreira GR, Cardozo LE, et al (2018) CEMiTool: a Bioconductor package for performing comprehensive modular co-expression analyses. BMC Bioinformatics 19:56.

https://doi.org/10.1186/s12859-018-2053-1

Shannon P (2003) Cytoscape: A Software Environment for Integrated Models of Biomolecular Interaction Networks. Genome Res 13:2498-2504. https://doi.org/10.1101/gr.1239303

Sneyers F, Rosa N, Bultynck G (2020) Type 3 IP3 receptors driving oncogenesis. Cell Calcium 86:102141. https://doi.org/10.1016/j.ceca.2019.102141 
Su Z, Yang Z, Xu Y, et al (2015) Apoptosis, autophagy, necroptosis, and cancer metastasis. Mol Cancer 14:48. https://doi.org/10.1186/s12943-015-0321-5

Szklarczyk D, Franceschini A, Wyder S, et al (2015) STRING v10: protein-protein interaction networks, integrated over the tree of life. Nucleic Acids Res 43:D447-D452. https://doi.org/10.1093/nar/gku1003

Tan F, Zhu H, Tao Y, et al (2015) Neuron navigator 2 overexpression indicates poor prognosis of colorectal cancer and promotes invasion through the SSH1L/cofilin-1 pathway. J Exp Clin Cancer Res 34:117. https://doi.org/10.1186/s13046-015-0237-3

Tenan M, Aurrand-Lions M, Widmer V, et al (2009) Cooperative expression of junctional adhesion molecule-C and -B supports growth and invasion of glioma. Glia NA-NA. https://doi.org/10.1002/glia.20941

Wang L, Yao L, Zheng Y-Z, et al (2015a) Expression of autophagy-related proteins ATG5 and FIP200 predicts favorable disease-free survival in patients with breast cancer. Biochem Biophys Res Commun 458:816-822. https://doi.org/10.1016/j.bbrc.2015.02.037

Wang M, Liu Y, Zou J, et al (2015b) Transcriptional co-activator TAZ sustains proliferation and tumorigenicity of neuroblastoma by targeting CTGF and PDGF- $\beta$. Oncotarget 6:9517-9530. https://doi.org/10.18632/oncotarget.3367

Warde-Farley D, Donaldson SL, Comes O, et al (2010) The GeneMANIA prediction server: biological network integration for gene prioritization and predicting gene function. Nucleic Acids Res 38:W214W220. https://doi.org/10.1093/nar/gkq537

Wright KL, Adams JR, Liu JC, et al (2015) Ras Signaling Is a Key Determinant for Metastatic Dissemination and Poor Survival of Luminal Breast Cancer Patients. Cancer Res 75:4960-4972. https://doi.org/10.1158/0008-5472.CAN-14-2992

Xu S, Wang P, Zhang J, et al (2019) Ai-IncRNA EGOT enhancing autophagy sensitizes paclitaxel cytotoxicity via upregulation of ITPR1 expression by RNA-RNA and RNA-protein interactions in human cancer. Mol Cancer 18:89. https://doi.org/10.1186/s12943-019-1017-z

Yan J, Zhao Q, Gabrusiewicz K, et al (2019) FGL2 promotes tumor progression in the CNS by suppressing CD103+ dendritic cell differentiation. Nat Commun 10:448. https://doi.org/10.1038/s41467-018-08271-x

Yao M, Zhou X, Zhou J, et al (2018) PCGF5 is required for neural differentiation of embryonic stem cells. Nat Commun 9:1463. https://doi.org/10.1038/s41467-018-03781-0

Yu X, Feng L, Liu D, et al (2016) Quantitative proteomics reveals the novel co-expression signatures in early brain development for prognosis of glioblastoma multiforme. Oncotarget 7:14161-14171. https://doi.org/10.18632/oncotarget.7416 


\section{Figures}

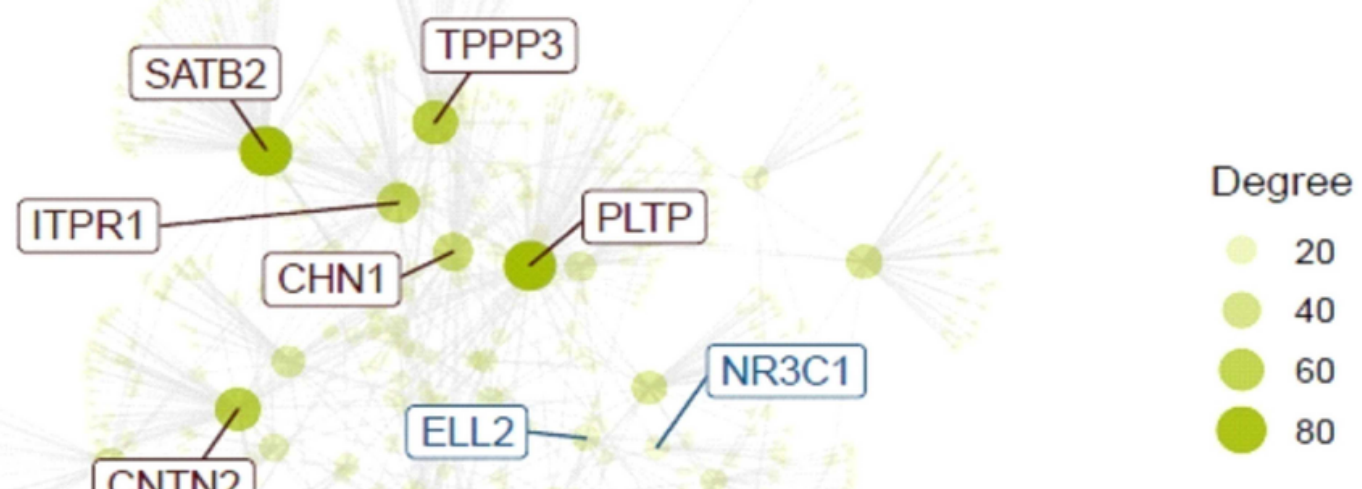

Hub

$\begin{array}{ll}\text { TFAP2A } & \text { a Co-expression } \\ & \text { a Interaction }\end{array}$

TFAP2A
a Interaction

NFAM1

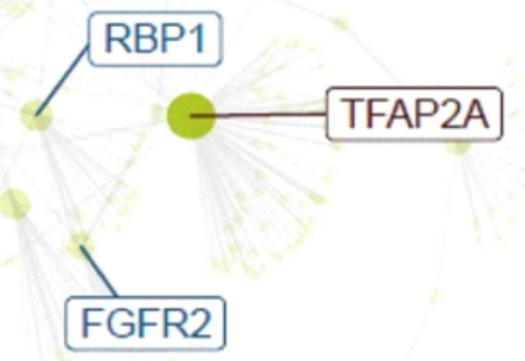

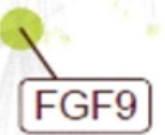

\section{Figure 1}

Gene networks of co-expression module displaying the most connected genes (hub genes). Analysis of co-expression reveal ITPR1 as hub gene in Group 3-MBs. The size of the node is proportional to its degree. The genes with co-expression are represented in blue and the genes with interactions are written in red. The module was constructed by CEMiTool package 

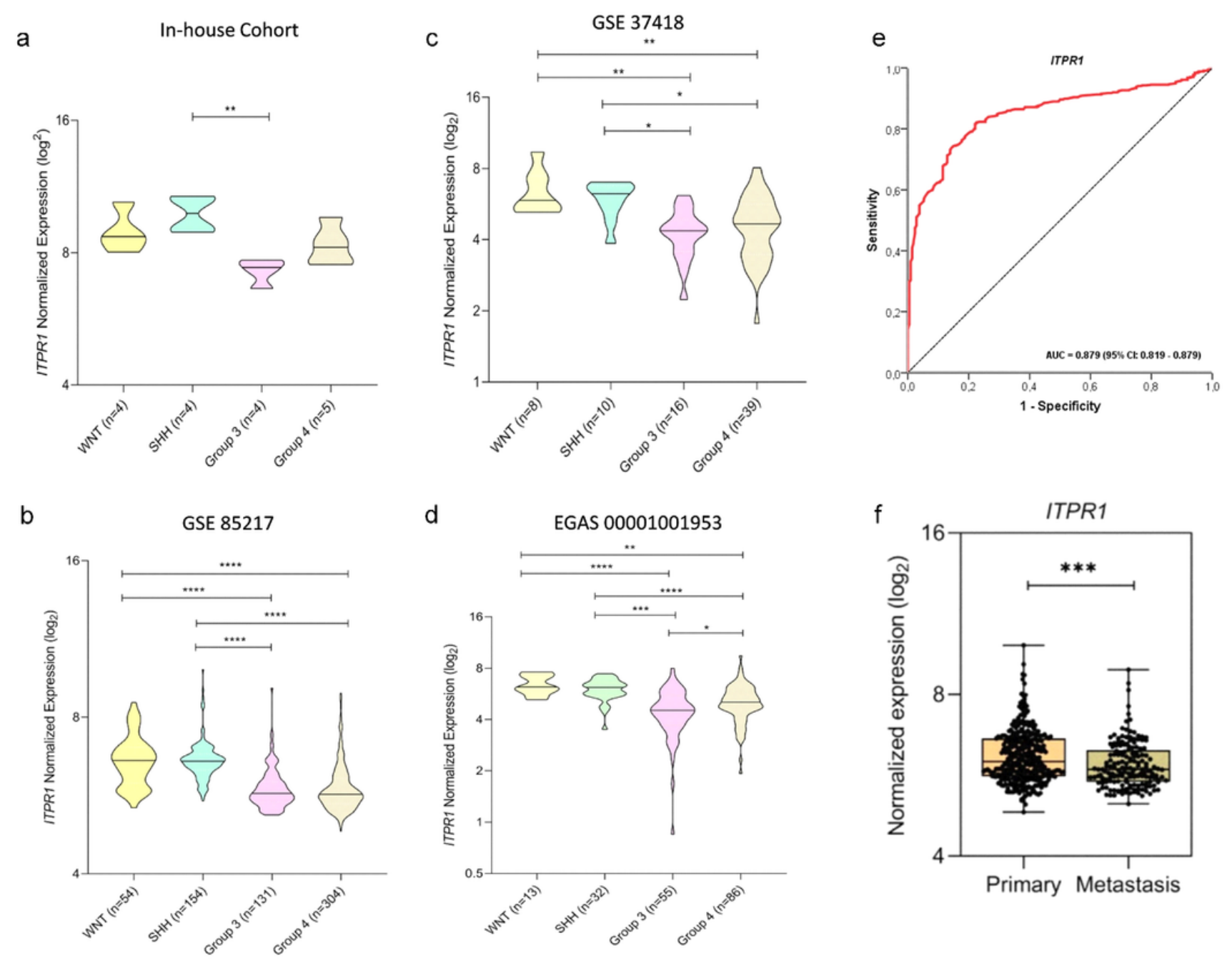

\section{Figure 2}

Overview of expression ITPR1 gene in MBs subtypes and clinical relevance. Violin plots showing the levels of ITPR1 gene expression among our RNA-seq data and three datasets of pediatric MBs samples obtained of R2 Genomic Analysis online platform. All analyzes were performed by separating the samples into molecular subgroups of MBs: (a) Validation of low levels of ITPR1 gene expression in our cohort of pediatric MB patients by RNA-Seq represented through the violin plots among the molecular subgroups of MBs (WNT n=4; SHH $n=4$; Group $3 n=4$ and Group 4 n=5); (b) GSE85217(n=643), (c) GSE37418 ( $n=73)$ and (d) EGAS00001001953 ( $n=186)$. The profile of ITPR1 gene expression was significantly observed downregulated in Grp3/Grp4-MBs when compared with others molecular subgroups of MB. ANOVA were conducted using the Tukey multiple comparisons post test to assess the statistical significance of the differences. * indicates $(p<0.001)$. (e) ROC curve analysis for prediction of G3 and G4 MBs among pediatric patients by ITPR1 level. (f) Low levels of ITPR1 are associated with metastasis in MBs samples primary $(n=343)$ and metastasis $(n=167),(p<0.05)$ 


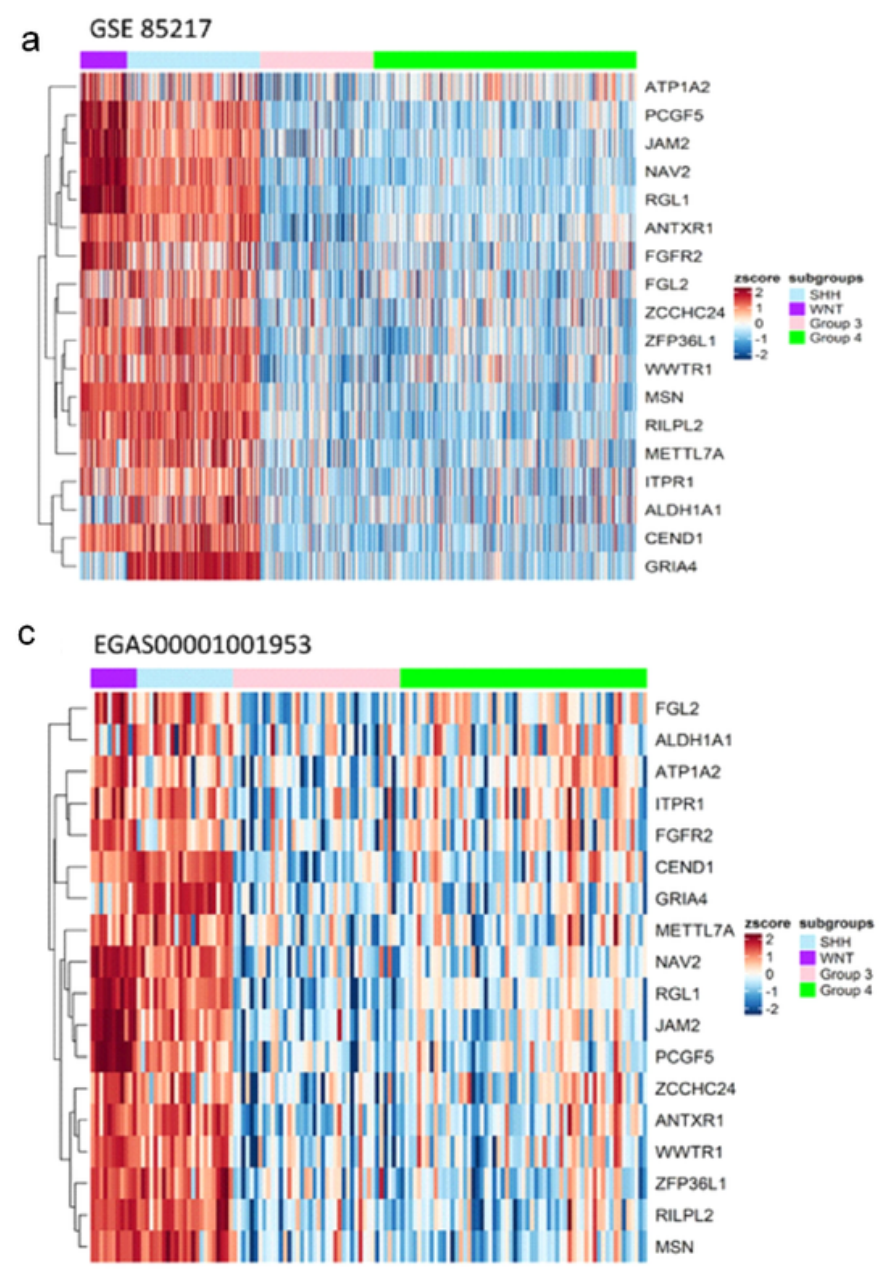

b GSE 37418

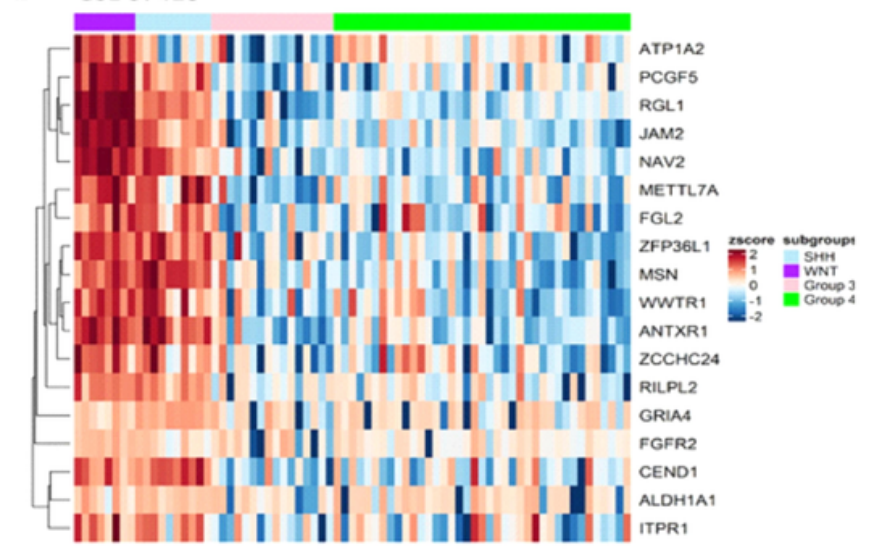

d Validation Cohort

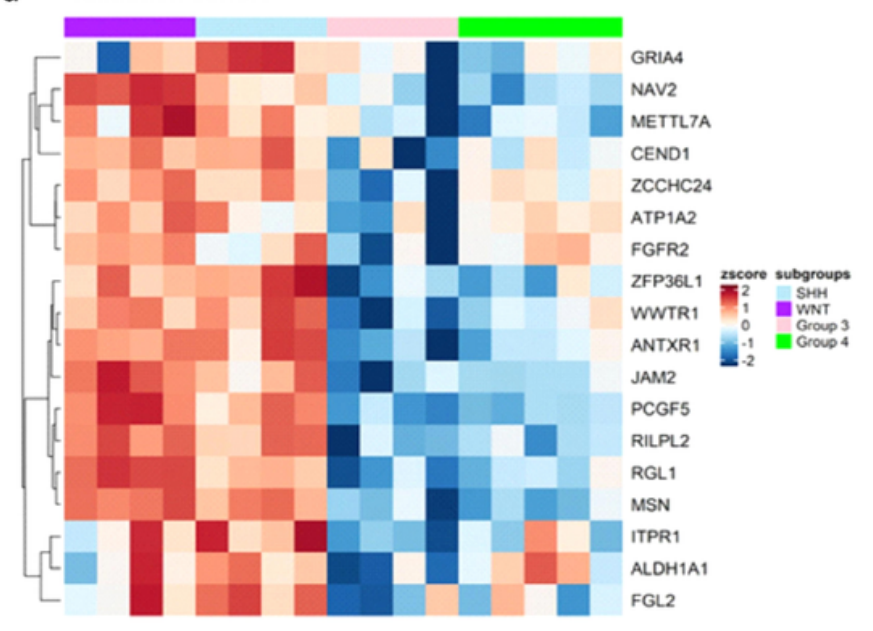

Figure 3

Hierarchical unsupervised clustering of primary MB datasets. Samples were clustered according molecular subgroups of MBs: SHH (blue), WNT (purple), Group 3 (pink) and Group 4 (green). Heatmaps summarizing a downregulated profile of expression genes positively coregulated with ITPR1 (FGL2, ALDH1A, ATP1A2, FGFR2, CEND1, GR1A4, METTL7A, NAV2, RGL1, JAM2, PCGF5, ZCCH24, ANTXR1, WWTR1, ZFP36L1, RILPL2, MSN) in (a) our cohort ( $n=17)$; (b) GSE85217(n=643), (c) GSE37418( $n=73$ ) and (d) EGAS00001001953 ( $n=186)$. Red indicates that the expression of genes is relatively upregulated, blue indicates that expression of genes is relatively downregulated. The heatmap was generated using the ComplexHeatmap package and Pearson distance as Metric. Clustering using the Ward.D2 algorithm 
a

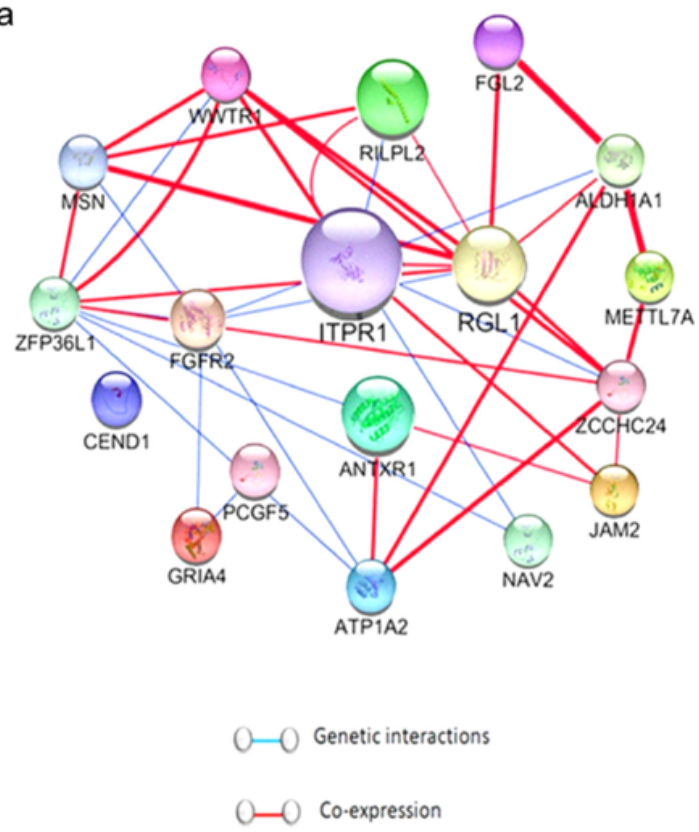

b

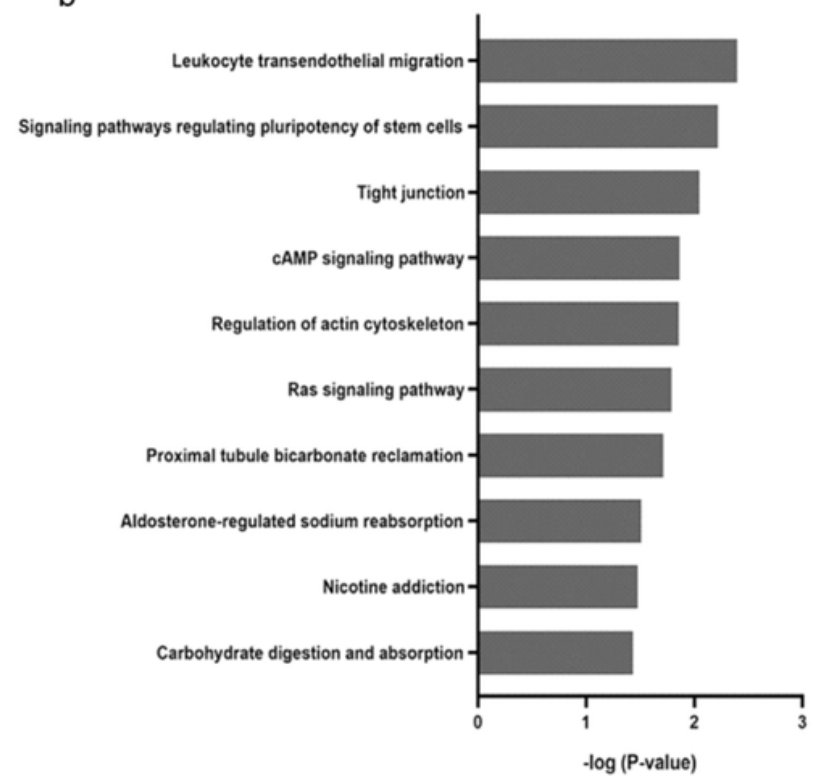

Figure 4

(a) PPI analysis reveals interactions potential of ITPR1 and its coregulated genes. Circles represent genes and the results within the circle represent the structure of proteins. The ITPR1-RGL1-ANTXR1 axisregulated indicated interaction of genes associated with worse prognosis. ITPR1-ATP1A2-MTTL7A-RGLI suggests a putative gene interaction coregulated with metastatic risk potential in Group 3-MBs. Lines colors represent evidence of the interaction of proteins between genes: genetic interactions (line in blue) and co-expression (line in red). (b) KEGG pathway Enrichment analysis in MBs: Abbreviations: KEGG, Kyoto Encyclopedia of Genes and Genomes. The significant enriched KEGG terms in MBs were based on their functions. Enrichment was done by Enrichr, using KEGG databases. The grayscale bars represent the $\mathrm{p}$-value, increased from the bottom up 
a

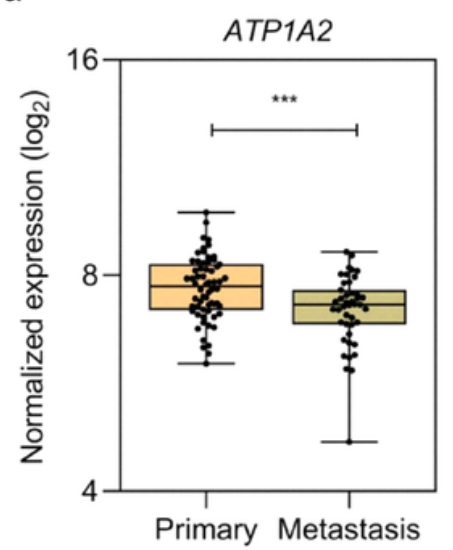

b

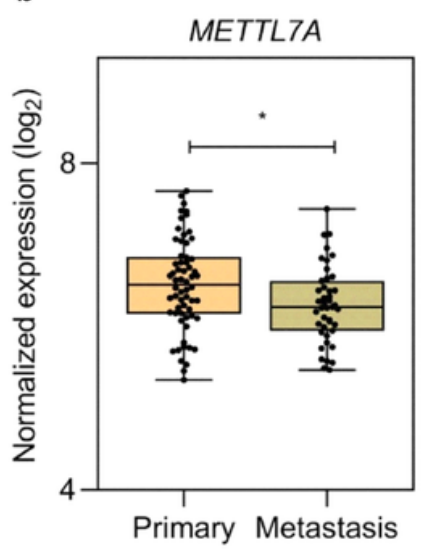

C

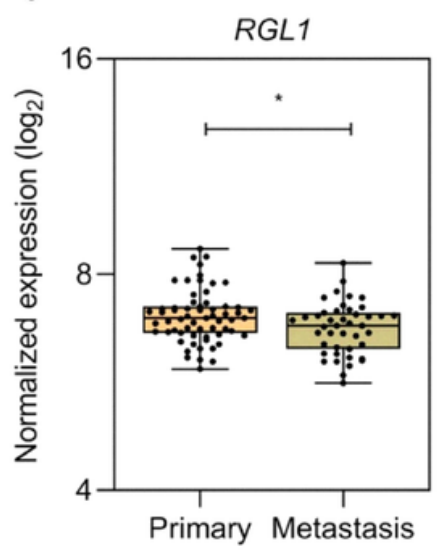

d

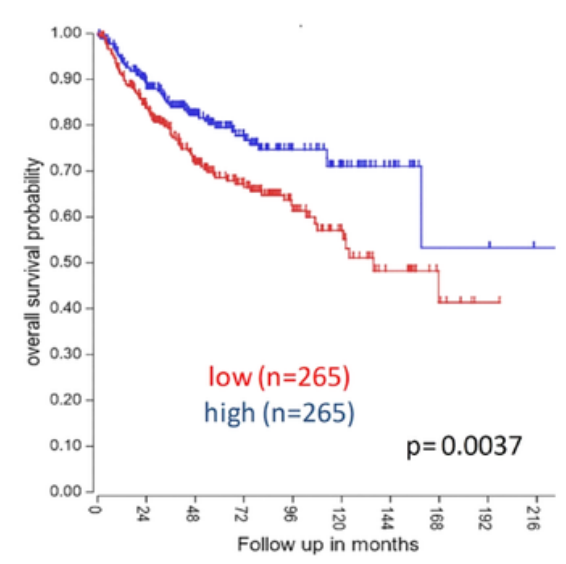

e

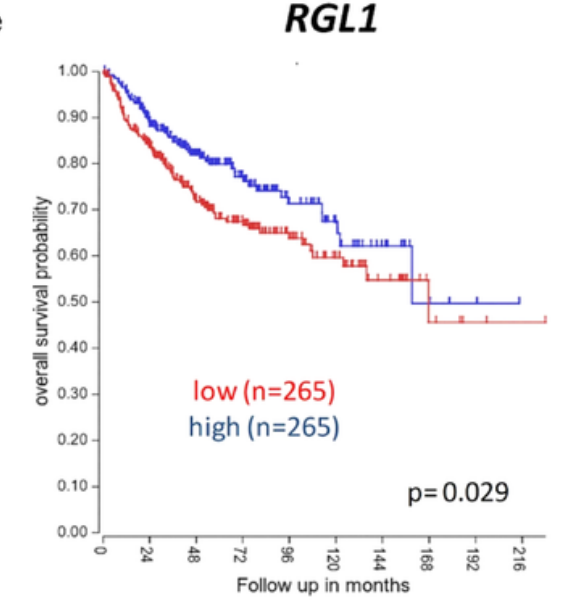

\section{Figure 5}

Analysis of ITPR1-gene expression with biological and clinical importance in MBs samples by GSE85217: The expression levels level of (a) ATP1A2, (b) MTTL7A and (c) RGL1 in Group 3-MBs primary samples $(n=62)$ compared to metastasis patients $(n=43)(p<0$.05). Kplan-Meier survival plots showing that lowexpression of the (d) ANTXR1 and (e) RGL1 reveals statistical significance associated with poor survival in MBs patients $p=0.0037$ and 0.029 respectively. Red, low-risk group, blue high-risk group. X-axis, time (months); Y-axis, overall survival probability.

\section{Supplementary Files}

This is a list of supplementary files associated with this preprint. Click to download.

- SupplementaryFig.1reviewed.pdf 
- SupplementaryFig.2reviewed.pdf

- SupplementaryFig.3reviewed.pdf

- SupplementaryTable01 reviewed.pdf

- SupplementaryTable02reviewed.pdf

- SupplementaryTable03reviewed.pdf 